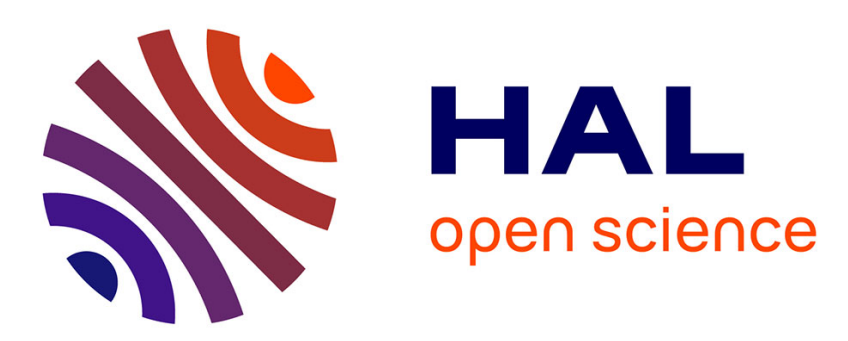

\title{
Biomechanical characterization of ex vivo human brain using ultrasound shear wave spectroscopy
} \author{
Emmanuel Nicolas, Samuel Calle, Stéphane Nicolle, David Mitton,
} Jean-Pierre Remenieras

\section{- To cite this version:}

Emmanuel Nicolas, Samuel Calle, Stéphane Nicolle, David Mitton, Jean-Pierre Remenieras. Biomechanical characterization of ex vivo human brain using ultrasound shear wave spectroscopy. Ultrasonics, 2018, 84, pp.119-125. 10.1016/j.ultras.2017.10.009 . hal-01652774

\section{HAL Id: hal-01652774 \\ https://hal.science/hal-01652774}

Submitted on 30 Nov 2017

HAL is a multi-disciplinary open access archive for the deposit and dissemination of scientific research documents, whether they are published or not. The documents may come from teaching and research institutions in France or abroad, or from public or private research centers.
L'archive ouverte pluridisciplinaire HAL, est destinée au dépôt et à la diffusion de documents scientifiques de niveau recherche, publiés ou non, émanant des établissements d'enseignement et de recherche français ou étrangers, des laboratoires publics ou privés. 


\title{
Biomechanical characterization of ex vivo human brain using ultrasound shear wave spectroscopy.
}

\author{
Emmanuel Nicolas ${ }^{\mathrm{a}, *}$, Samuel Callé ${ }^{\mathrm{a}}$, Stéphane Nicolle ${ }^{\mathrm{b}}$, David Mitton ${ }^{\mathrm{b}}$, Jean-Pierre Remenieras ${ }^{\mathrm{a}}$ \\ ${ }^{a}$ UMR Inserm U930, Université François-Rabelais, Tours, France \\ ${ }^{b}$ Université Claude Bernard Lyon 1, IFSTTAR, LBMC UMR T9406, F69622, Lyon, France
}

\begin{abstract}
The characterization of brain tissue is crucial to better understand neurological disorders. Mechanical characterization is an emerging tool in that field. The purpose of this work was to validate a transient ultrasound technique aimed at measuring dispersion of mechanical parameters of the brain tissue. The first part of this work was dedicated to the validation of that technique by comparing it with two proven rheology methods: a rotating plate rheometer, and a viscoelastic spectroscopy apparatus. Experiments were done on tissue mimicking gels. Results were compared on storage and loss modulus in the $20 \mathrm{~Hz}$ to $100 \mathrm{~Hz}$ band. Our method was validated for the measurement of storage modulus dispersion, with some reserves on the measurement of loss modulus. The second part of this work was the measurement of the mechanical characteristics of ex vivo human white matter. We were able to measure the dispersion of the storage and loss modulus in the $20 \mathrm{~Hz}$ to $100 \mathrm{~Hz}$ band, fitting the data with a custom power law model.
\end{abstract}

Keywords: ultrasound, shear wave elastography, high frequency rheology, brain

\section{Introduction}

Benefiting from the wide elasticity of human body tissues (eight orders of magnitude for the elastic modulus from soft to hard tissues), manual palpation is a common practice in medicine. However, this practice is more qualitative than quantitative and is limited to tissues to which we have direct access. It is well known that wave propagation within a tissue is related to its mechanical properties, and tools based on the principles of wave propagation, such as elastography, may be advantageous for quantitative measurement of the mechanical features of tissues. Elastography could then be seen as a quantitative palpation device, capable of better discrimination of tissues and variations within them.

Brain elastography has been recognized as a promising diagnostic tool (Vappou et al., 2008; Kruse et al., 2008; Sack et al., 2008; Weaver et al., 2012). Neurodegenerative diseases such as Alzheimer's disease are difficult to evaluate by conventional imaging methods. These diseases have diffuse effects on the brain, slowly

${ }^{*}$ Corresponding author at: Inserm U930 - Équipe 5, UFR de Médecine, 10 Boulevard Tonnellé, B.P. 3223, 37032 Tours Cedex 1, France

Email address: emmanuel@nicolas.sh (Emmanuel Nicolas) replacing normal tissue with plaques and neurofibrillary tangles. It is assumed that such changes affect the mechanical properties of the brain (Huston et al., 2011). It is also theorized that other diseases, such as normal pressure hydrocephalus,result in change in brain mechanical properties (Bradley Jr. et al., 1991).

We propose here preliminary results of shear wave speed dispersion measurement using ultrasound transient elastography applied to calibrated viscoelasticity phantoms and to the human brain ex vivo.

\section{Interest of measuring viscoelastic parameters dis- persion for medical applications}

Theoretical and physical factors indicate that the presence of microscopic obstacles may influence not only the absolute value of viscoelastic tissue parameters, but also their relationship with frequency (?). There is a physical link between the propagation of shear waves in a viscoelastic medium and its viscoelastic properties (Szabo and Wu, 2000; Sandrin et al., 2004; Catheline et al., 2004). In particular, we know that the linear dynamic shear modulus of biological soft tissues appears to be proportional to the fractional power of frequency, i.e. the frequency-dependent modulus may be 
modeled by a power law, the exponent parameter representing a mechanical property inherent to a given tissue (Bagley, 1983; Schiessel et al., 1995; Tanter et al., 2006; Nicolle et al., 2013). This behavior cannot be modeled by the classical Voigt or Maxwell model widely used in elastography. Indeed, the Voigt model predicts a frequency-independent elastic modulus $G^{\prime}(\omega)$ and a loss modulus $G^{\prime \prime}(\omega) \propto \omega$ that is directly proportional to the angular frequency, which is in obvious contradiction with fractional law behavior. In contrast, the Maxwell model allows dispersion for both moduli but not according to a power law with equal exponents for $G^{\prime}(\omega)$ and $G^{\prime \prime}(\omega)$. There are, however, rheological models that are capable of describing power law behavior for the complex shear modulus, named springpot models (Bagley, 1983; Schiessel et al., 1995; Tanter et al., 2006). A basic springpot model is equivalent to a hierarchical organization of springs and dashpots (Schiessel et al., 1995) and can continuously interpolate between a purely solid material $(\gamma=0)$ and a purely liquid $(\gamma=1)$ material with respect to the power law exponent for $G^{*}(\omega)=G^{\prime}(\omega)+i G^{\prime \prime}(\omega) \propto(i \omega)^{\gamma}$. However, this model has limitations; the power exponent $\gamma$ is restricted to the interval $[0,1]$, and second the ratio $\frac{G^{\prime \prime}(\omega)}{G^{\prime}(\omega)}$ is linked to the power exponent via $\frac{G^{\prime \prime}(\omega)}{G^{\prime}(\omega)}=\tan \left(\frac{\gamma \pi}{2}\right)$ with the same $\gamma$.

The dispersion of the shear modulus has been studied in several types of tissue, using different approaches to model such dispersion. Results obtained in breast tumors, brain tissue and the liver are briefly presented below. It was shown by MRE by Tanter et al. (2006) in in vivo breast carcinoma that the frequency behavior of $G^{\prime}(\omega)$ (storage modulus) and $G^{\prime \prime}(\omega)$ (loss modulus) are well modeled by a non-integer power law: $G^{\prime}(\omega)=\omega^{\gamma}$, $G^{\prime \prime}(\omega)=\omega^{\gamma}$ with $\gamma=0.75$. In this set of in vivo breast data, although the data ratio is not frequency dependent, its value corresponds to the ratio predicted by a spring pot model $\frac{G^{\prime \prime}(\omega)}{G^{\prime}(\omega)}=\tan \left(\frac{\beta \pi}{2}\right)$ with a constant $\beta=0.15$ different to and lower than $\gamma$. To simulate this specific soft tissue architecture, a new rheological model was introduced by the authors based on a network with an infinite ladder of spring pots. The basic element and the network have the same nature, i.e. the basic element parameter and the network element parameter are the same. In this new rheological model, the frequency behavior in $\omega^{\gamma}$ of the dynamic and loss moduli is due to the spring pot corresponding to the basic element of the network. Thus, it characterizes the cells. The ratio $\frac{G^{\prime \prime}(\omega)}{G^{\prime}(\omega)}=\tan \left(\frac{\beta \pi}{2}\right)$ is due to the network and the arrangements of the spring pots. The $\beta$ parameter is different from $\gamma$ and its value is between 0 and 1. For solid ma- terial, the $\beta$ parameter is close to 0 , while for liquid material it is closer to 1 . Moreover, carcinoma is characterized by a high $\beta$ value, corresponding to a liquid-like behavior, while a fibro adenoma is characterized by a low $\beta$ value corresponding to solid-like behavior. Thus, the rheological model can be adapted to the soft tissue structure.

Undertaking a multiple frequency study using MRE involves making multiple measurements at each frequency, which can considerably lengthen the examination time. With ultrasound elastography, a short, wide band excitation signal can be used which will allow a dispersion study using a single acquisition, or even multiple acquisitions with varying center frequencies to cover a wider band.

In this study we tested the feasibility of measuring the dispersion of mechanical properties using transient shear wave propagation in a tissue mimicking phantom and in the human brain ex vivo.

\section{Materials and methods}

\subsection{Experimental setup}

The experimental setup used in this study and presented schematically in figure 1 is based on previous work by Catheline et al. (2004). A purely transversally polarized plane wave propagating in the $x$ direction is mechanically induced in the studied medium. A multi-element linear ultrasound probe (128 elements, $2.8 \mathrm{MHz}$ center frequency, Vermon, France) was placed perpendicularly to the direction of propagation, to allow observation of the wave propagation. The probe was connected to an ultrafast echographic scanner (Aixplorer, SuperSonic Imagine, France), capable of acquiring 5000 frames per second, used to measure displacement inside the medium. On each acquisition, the scanner triggered a signal generator (Tektronix AFG 31023), driving a vibrator (Bruel \& Kjaer 4826). A rigid Plexiglas plate was connected to the vibrator generating a sum of plane, low amplitude shear waves in the medium being tested. This experimental setup is represented in figure 2 .

The signal used to drive the vibrator was a Gaussian shaped wave, providing a single, broadband acquisition $(20 \mathrm{~Hz}$ to $150 \mathrm{~Hz})$.

To validate our method and compare the measurements, two classical material characterization methods were used, the first using hyper-frequency viscoelastic spectroscopy (Hadj Henni et al., 2011) (Rheospectris, Rheolution, Canada), with a cylindrical sample (10 mm diameter, $45 \mathrm{~mm}$ height). This method allows exploration of both $G^{\prime}$ and $G^{\prime \prime}$ in a wide frequency range 


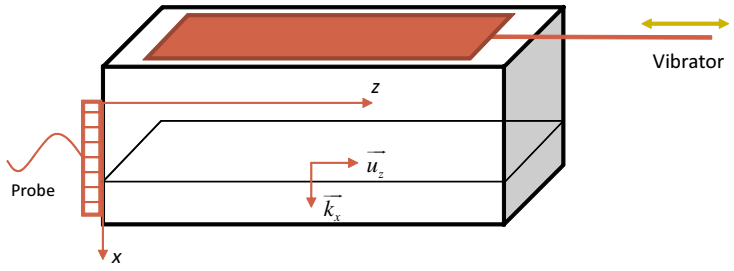

Figure 1: Representation of the experiment. The plate moves along the horizontal axis ( $z$ axis). The probe is placed perpendicular to it, along the $x$ axis.

$(10 \mathrm{~Hz}$ to $1000 \mathrm{~Hz})$. The second was a high-frequency rheometer using a thin disk-shaped sample $(15 \mathrm{~mm}$ diameter, sub-millimetric thickness). This method was used to measure $G^{\prime}$ and $G^{\prime \prime}$ between $10 \mathrm{~Hz}$ and $500 \mathrm{~Hz}$.

The phantoms used were made of a mixture of a triblock SEBS copolymer (Kraton Polymers, Univar, France) dissolved in white mineral oil (Oudry et al., 2009). The scatterers used were silica powder with grain size ranging from $35 \mu \mathrm{m}$ to $70 \mu \mathrm{m}$. These types of phantom present several advantages over more classical types (agar gelatin), in particular excellent mechanical resistance and stability of the mechanical parameters (tested over 18 months). The two variable parameters were the oil kinematic viscosity and the polymer concentration. These parameters provide a wide range of mechanical parameters, with a Young's modulus of $2.2 \mathrm{kPa}$ to $150 \mathrm{kPa}$ and an attenuation coefficient of $0.1 \mathrm{~dB} \mathrm{~cm}^{-1}$ to $40 \mathrm{~dB} \mathrm{~cm}^{-1}$.

The phantom used in elastography experiments was of cylindrical shape, $15 \mathrm{~cm}$ high, $15 \mathrm{~cm}$ diameter. The homogeneity of the phantoms was verified from a structural point using B-mode ultrasound imaging and confirmed by Magnetic Resonance Elastography (MRE) (figure 3).

\subsection{Data processing}

The data obtained from the scanner was raw demodulated in-phase and quadrature components (complex IQ data). The IQ signal can be written:

$$
I Q(x, z, n)=I(x, z, n)+j Q(x, z, n)=A(x, z, n) e^{j \phi(x, z, n)}
$$

where $A$ is the signal amplitude and $\phi(x, z, n)$ the signal phase at position $(x, z)$ for the $n^{\text {th }}$ pulse. The velocity estimator is defined in equation 2 , an extension of the autocorrelation estimator developed by Hoeks et al. (1994). This estimates the mean velocity using multiple, spatially contiguous IQ sample volumes in time.
This approach increases the accuracy of the velocity estimated at the expense of decreasing the spatial resolution, expressed mathematically as:

$$
\begin{gathered}
V(x, z, t)=\frac{\lambda}{4 \pi T} \times \\
\arg \left(\sum_{a=0}^{N_{a}} \sum_{b=0}^{N_{b}} I Q(x, z-a, t-b) \overline{I Q}(x, z-a, t-b-1)\right)
\end{gathered}
$$

where $\lambda$ is the wavelength and $T$ the time interval between two acquisitions. $N_{a}$ is the number of samples defining the volume of interest and $N_{b}$ the number of temporal samples in the autocorrelation over which the mean velocity is estimated.

The axial resolution obtained was $1 \lambda=0.5 \mathrm{~mm}$. For each subsample volume at a given depth, the tissue velocity was estimated with an observation window of 8 samples with $50 \%$ overlap, giving a temporal resolution of $0.4 \mathrm{~ms}$.

Figures 4 and 5 illustrate the tissue velocity calculated within a phantom. In these figures the purely transversal, shear nature of the wave can be easily seen and the amplitude decreasing with depth $x$ is clearly visible. In particular figure 5 shows the shear wave propagation in a time space domain for a given $z$, and the group speed of the shear wave can be approximated from that visualization. The rebound of the wave can also be seen (vertically symmetric wave starting at the bottom, at $60 \mathrm{~ms}$ ).

In this configuration, assuming that the media being tested was homogeneous, isotropic, viscoelastic and incompressible, the shear wave generated by the shaking plate was considered to be purely transverse. Thereafter the velocity field could be expressed by the following Helmholtz wave equation:

$$
G^{*}(\omega) \frac{\partial^{2} V_{z}(x)}{\partial x^{2}}+\rho \omega^{2} V_{z}(x)=0
$$

with $\omega$ the angular frequency and $G^{*}(\omega)$ the complex shear modulus. The amplitude of the velocity which solved eq. 3 for a given frequency was:

$$
V_{z}\left(\omega_{f}, x\right)=V_{0}\left(\omega_{f}\right) \mathrm{e}^{j k^{\prime}\left(\omega_{f}\right) x} \mathrm{e}^{-\alpha\left(\omega_{f}\right) x}
$$

with $\omega_{f}$ a fixed frequency, $k$ the complex wave number, $k^{\prime}$ the real part of the wave number and $\alpha$ the imaginary part (attenuation coefficient), both being frequency-dependent. 

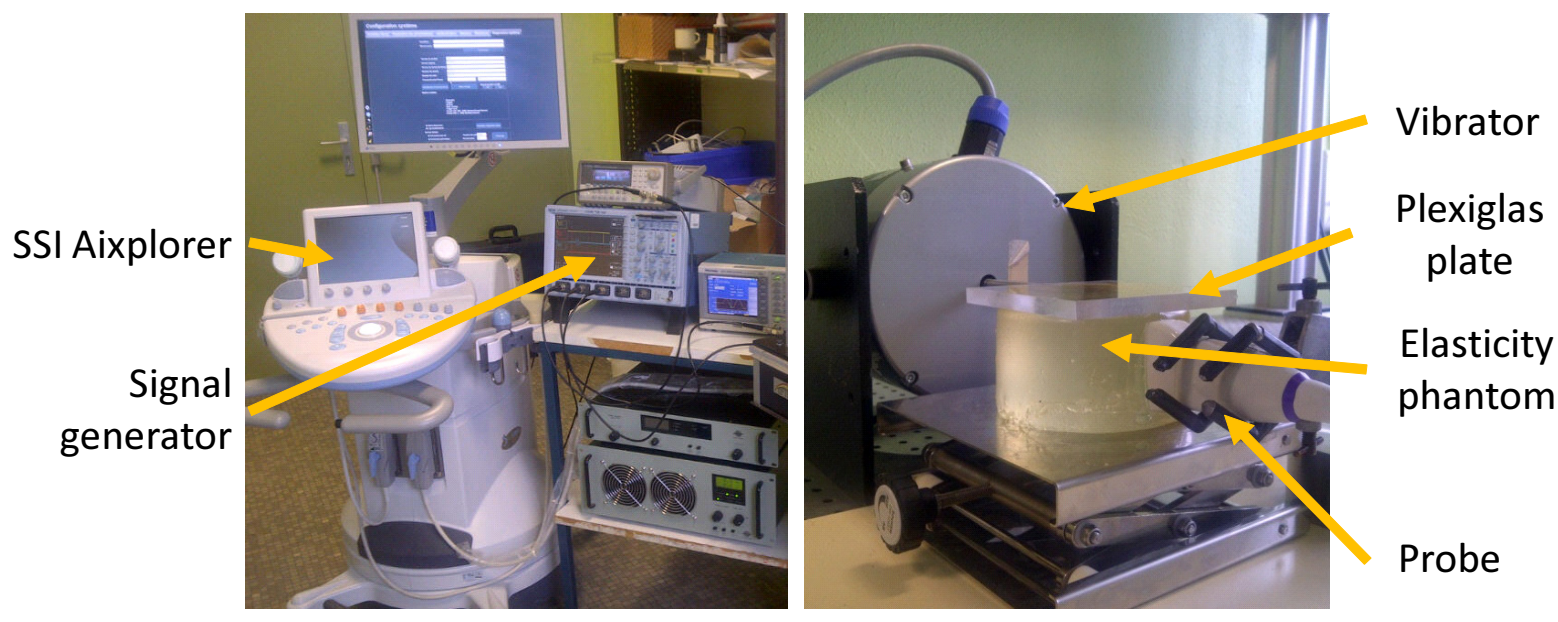

Figure 2: Experimental setup

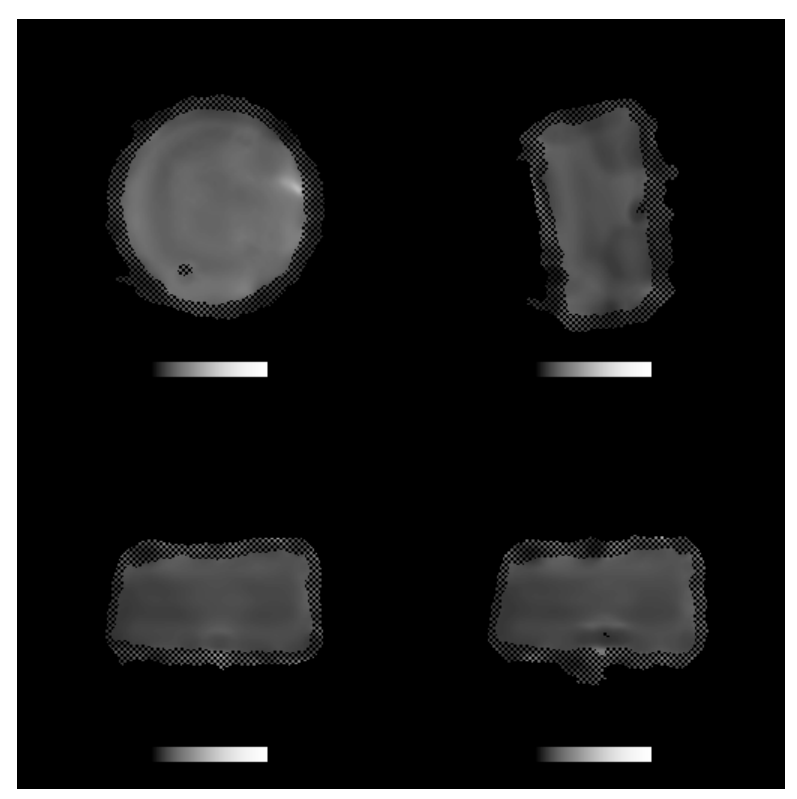

Figure 3: Elastogram obtained for a phantom using a commercial sequence on a Siemens 3T MRI. Four axes of view are shown. The data are represented as a mechanical index, representative of the material stiffness. The black mesh on the edges of the phantom is indicative of a low confidence index.
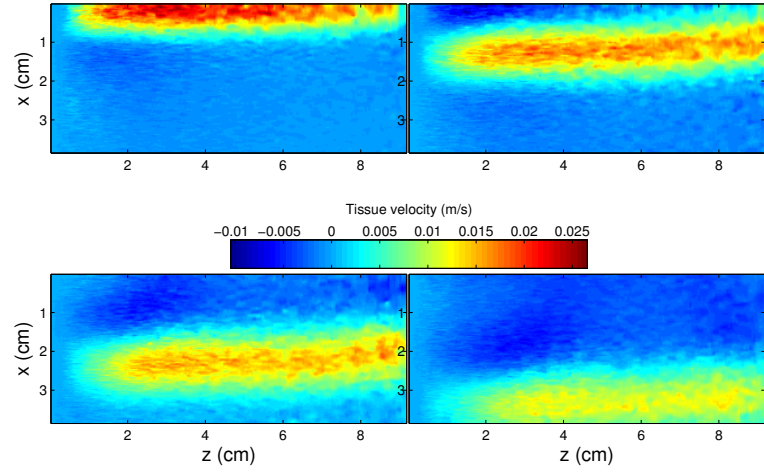

Figure 4: Particle velocity field at $20 \mathrm{~ms}, 30 \mathrm{~ms}, 40 \mathrm{~ms}$ and $50 \mathrm{~ms}$ after excitation. This confirms the plane shear nature of the wave. Attenuation due to viscosity can also be observed.

If $U_{z}(\omega, x)$ is the complex spectral amplitude of $V_{z}(\omega, x)$, computed by temporal Fourier transform, the inverse-problem is solved in the frequency space domain for a fixed $\omega_{f}$. The following two expressions could then be used to estimate velocity and attenuation, respectively:

$$
\begin{aligned}
& A_{z}(k)=\left|\operatorname{FFT}_{x}\left(U_{z}\left(\omega_{f}, x\right)\right)\right| \\
& B_{z}(x)=\ln \left(\left|U_{z}\left(\omega_{f}, x\right)\right|\right)
\end{aligned}
$$

with $\mathrm{FFT}_{x}$ the spatial Fourier transform.

The maximum of $A_{z}$ provides $k_{x_{\max }}$, the wave number associated with the maximum amplitude of the wave field at frequency $\omega_{f}$. An example of this is shown in 


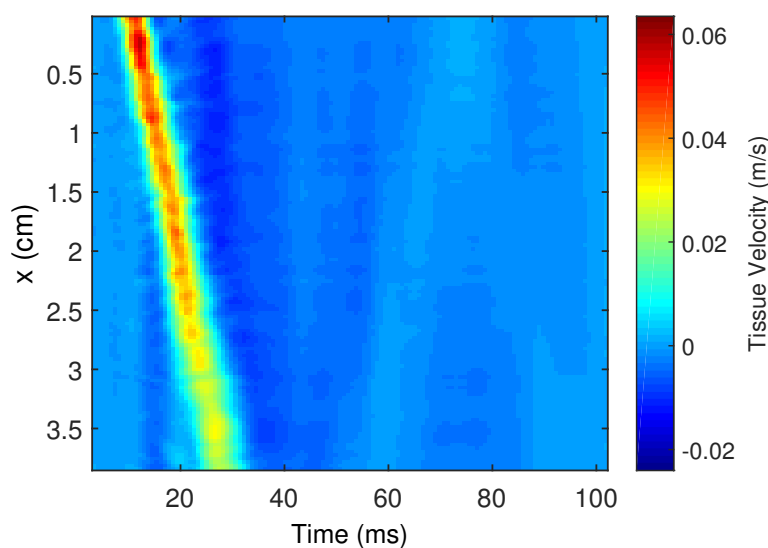

Figure 5: Particle velocity field for a given $z$. The slope of the curve provides a quick approximation of the group velocity.

figure 6 . The transverse wave velocity $c_{T}$ could then be deduced:

$$
c_{T}\left(\omega_{f}\right)=\frac{\omega_{f}}{2 \pi k_{x_{\max }}(\omega)}
$$

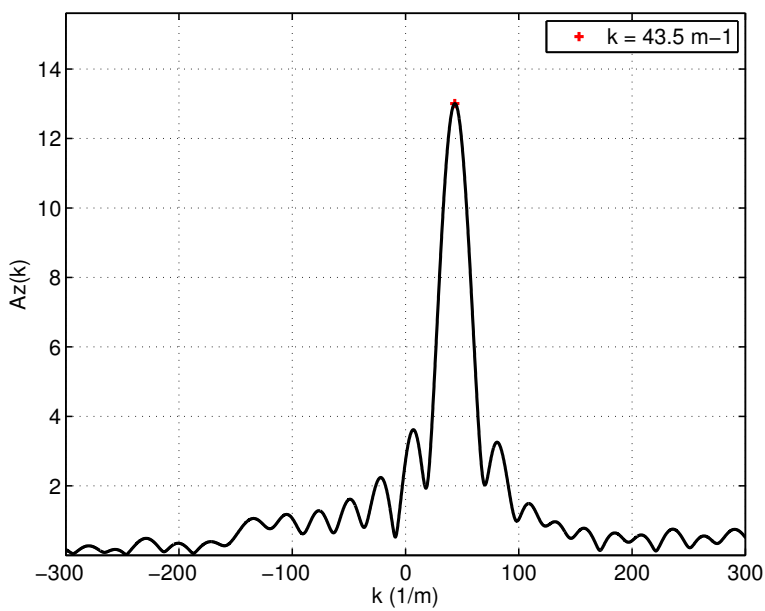

Figure 6: Example of $A_{z}(k)$, for $f=60 \mathrm{~Hz}$. In this example, $k=43.5 \mathrm{~m}^{-1}$, and $c_{T}=1.38 \mathrm{~m} \mathrm{~s}^{-1}$.

Linear regression of $B_{z}(x)$ provides $-\alpha$. An example is shown in figure 7.

In order to estimate the quality of the measurements by elastography, comparison with rheological measurements on a similar phantom was carried out. Without making hypotheses on the viscoelastic behavior of the material the complex shear modulus can be expressed:

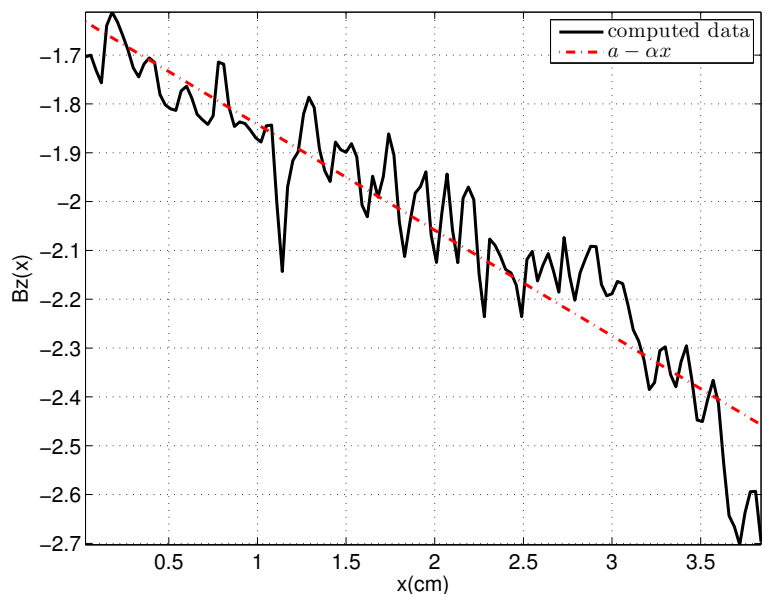

Figure 7: Example of $B_{z}(x)$, for $f=60 \mathrm{~Hz}$

$$
G^{*}(\omega)=\rho \frac{\omega^{2}}{k^{2}}
$$

Given that $G^{*}=G^{\prime}+j G^{\prime \prime}$ and $k=k^{\prime}+j \alpha$ (viscoelastic medium), from equation 8 it could finally be deduced that:

$$
\begin{aligned}
G^{\prime}(\omega) & =\rho \omega^{2} \frac{k^{\prime 2}-\alpha^{2}}{\left(k^{\prime 2}+\alpha^{2}\right)^{2}} \\
G^{\prime \prime}(\omega) & =-2 \rho \omega^{2} \frac{k^{\prime} \alpha}{\left(k^{\prime 2}+\alpha^{2}\right)^{2}}
\end{aligned}
$$

All these steps were repeated for each frequency in the $20 \mathrm{~Hz}$ to $120 \mathrm{~Hz}$ band, with $0.5 \mathrm{~Hz}$ resolution.

A typical acquisition consisted of 128 parallel echographic lines and enough frames to strobe the entire shear wave (usually 256 or 512) at 5000 frames per second. The acquisition depth was approximately $10 \mathrm{~cm}$, with $\Delta z=500 \mu \mathrm{m}$ and $\Delta x=300 \mu \mathrm{m}$. The calculations were repeated for every depth, indicating the robustness of the algorithm.

Viscoelastic parameters were estimated using a model developed by Nicolle et al. (2010). In this model, $\mathrm{G}$ is expressed as:

$$
G(i \omega)=K(i \omega)^{n}+i \eta_{\infty} \omega
$$

with $K$ the coefficient of consistence, $n$ the linear constitutive parameter and $\eta_{\infty}$ the viscosity at high frequency. 
The real and imaginary parts of $G$ are respectively:

$$
\begin{aligned}
G^{\prime}(\omega) & =K \cos \left(\frac{\pi}{2} n\right) \omega^{n} \\
G^{\prime \prime}(\omega) & =K \sin \left(\frac{\pi}{2} n\right) \omega^{n}+\eta_{\infty} \omega
\end{aligned}
$$

\section{Results}

\subsection{Calibrated, homogeneous phantoms}

The experiment was conducted on two different gels, the first with a stiffness and sound velocity close to those of brain tissue, and the second with a higher Young's modulus. Their characteristics are presented in table 1.

To compare the three methods, three phantoms were made (one for each method) in a single session to avoid differences in characteristics.

The results for the first phantom are presented in figure 8 . Figures $8 \mathrm{a}$ and $8 \mathrm{~b}$ represent $G^{\prime}$ and $G^{\prime \prime}$ for the three methods, respectively. The results for the second gel are presented in figure 9 . There was a a good match in the results between the three methods for the storage modulus. Moreover, the values of $G^{\prime}$ were in accordance with the estimated Young's modulus. The dispersion of the storage modulus was low, in accordance with the low viscosity components of the gel.

The loss modulus measurements were less reliable, the SWE technique being less reliable especially in gel 2 (see figure $9 b$ ).

Our method was based on a measurement of $k$ for a fixed $\omega_{f}$. Thus, when the wavelength was longer than the observation window, measurement was bound to be difficult. We followed the propagation of the shear wave in a window of $3.8 \mathrm{~cm}$ ( $x$ axis, the size of the probe) and a gross estimate of the shear wave speed could be made using $c_{s}=\sqrt{\frac{G^{\prime}}{\rho}}$, with $c_{s}$ the shear wave speed, and $\rho$ the density of the gel, measured at $1 \mathrm{~g} \mathrm{~cm}^{-3}$. For gel $1 G^{\prime} \approx 1 \mathrm{kPa}$, and for gel $2 G^{\prime} \approx 2.5 \mathrm{kPa}$ yielding $c_{s} \approx 1 \mathrm{~m} \mathrm{~s}^{-1}$ for gel 1 and $c_{s} \approx 1.5 \mathrm{~m} \mathrm{~s}^{-1}$ for gel 2 . This can explain the drop found in the value of $G^{\prime}$ for gel 2 (figure 9a). In that case, at $40 \mathrm{~Hz}$ we had a wavelength of about $\lambda=4 \mathrm{~cm}$, rendering evaluation of $k$ difficult. For gel 1 there was no drop, as the wavelength was shorter (about $2.5 \mathrm{~cm}$ ).

The evaluation of $G^{\prime \prime}$ was more challenging because we needed to assess the attenuation of the shear wave. For frequencies under $40 \mathrm{~Hz}$, there was a discrepancy between the SWE method and the other two methods for both gels. As before, estimating an attenuation on less than a wavelength was difficult. The tiniest field variation, whether noise related or due to a small heterogeneity has a significant effect on the measurement.
For frequencies above $40 \mathrm{~Hz}$ the results were correlated with the other methods. Adding more copolymer to the gel mixture tended to increase its shear viscosity, in accordance with the behavior observed at low frequencies by Oudry et al. (2009).

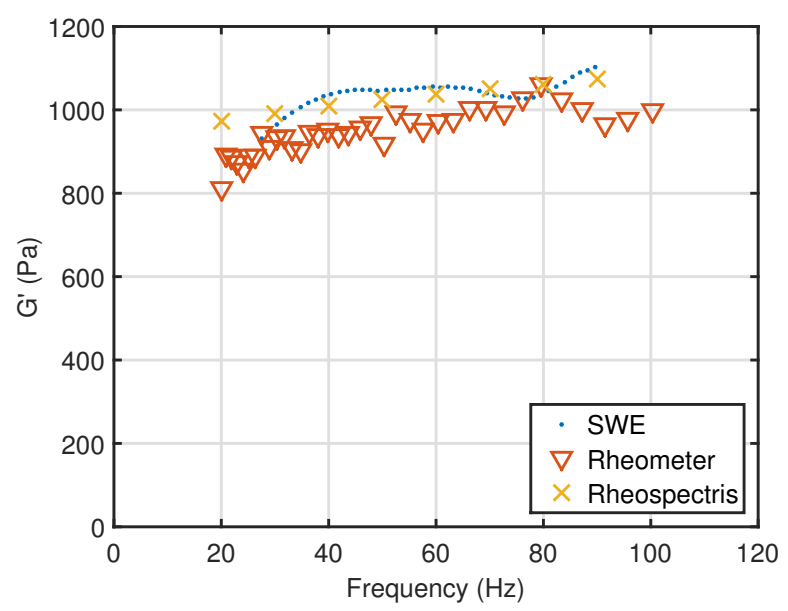

(a) Storage modulus

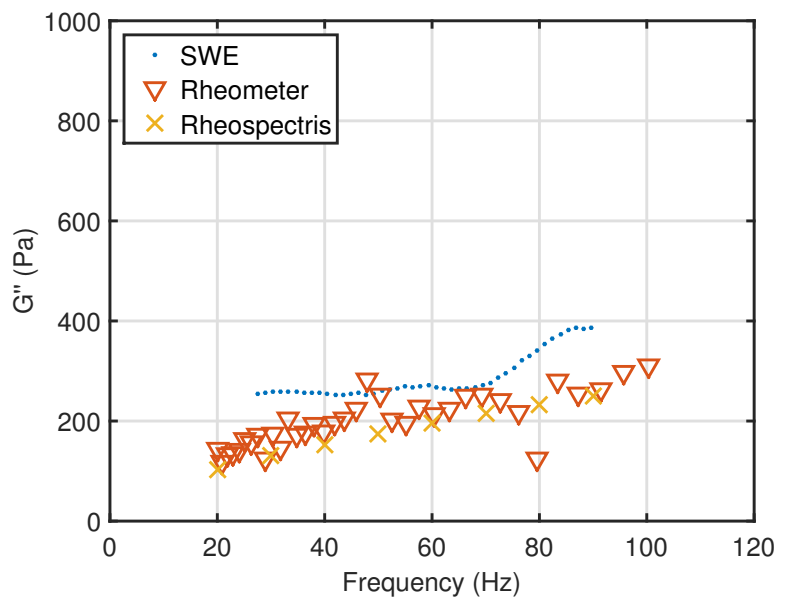

(b) Loss modulus

Figure 8: Real and imaginary parts of the complex shear modulus measured for gel 1 , made of oil at $35 \mathrm{~mm}^{2} \mathrm{~s}^{-1}$ mixed with $3.2 \%$ of copolymer. The three measurement methods are compared: Shear Wave Elastography (SWE), high-frequency rheometer, and hyperfrequency viscoelastic spectroscopy.

\subsection{Ex vivo brain}

The method was then tested on an ex vivo human brain, extracted 48 hours after death. The experimental setup similar to the previous setup, with the Plexiglas plate placed on top of the brain and the ultrasound probe placed across the parietal and temporal lobes, as seen in figure 11. In order to measure the mechanical characteristics on an homogeneous area, the measurement was 
Table 1: Gel characteristics. Young's modulus was estimated using the experimental law by Oudry et al. (2009)

\begin{tabular}{cccc}
\hline Phantom & $\begin{array}{c}\text { Oil viscosity } \\
\text { at } 20{ }^{\circ} \mathrm{C} \\
\left(\mathrm{mm}^{2} \mathrm{~s}^{-1}\right)\end{array}$ & $\begin{array}{c}\text { Copolymer } \\
\text { concentration } \\
\text { (percent of oil quantity) }\end{array}$ & $\begin{array}{c}\text { Estimated } \\
\text { Young's modulus } \\
(\mathrm{kPa})\end{array}$ \\
\hline 1 & 35 & 3.2 & 2.8 \\
2 & 35 & 5 & 8.4 \\
\hline
\end{tabular}

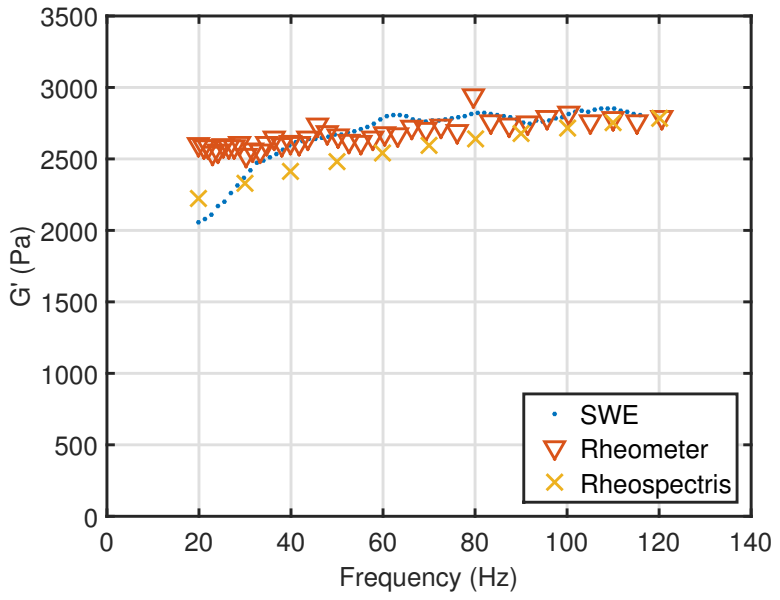

(a) Storage modulus

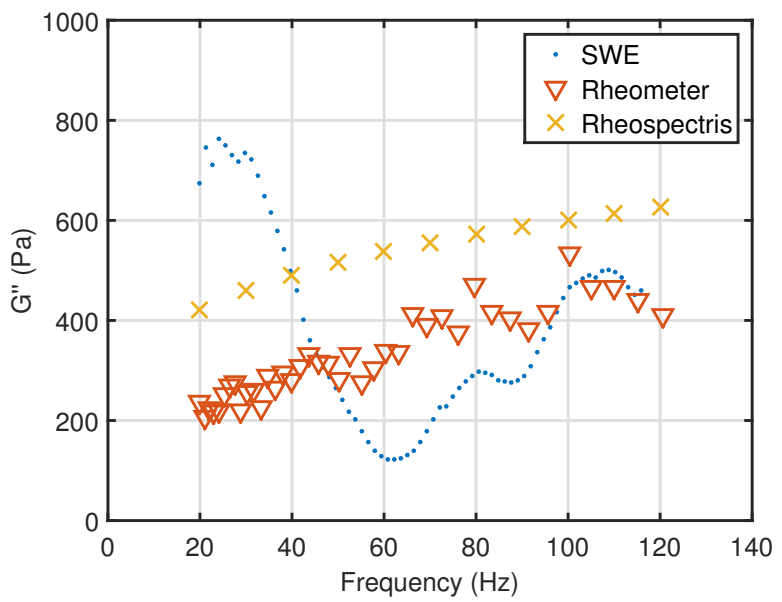

(b) Loss modulus

Figure 9: Real and imaginary parts of the complex shear modulus measured for gel 2 , made of oil at $35 \mathrm{~mm}^{2} \mathrm{~s}^{-1}$ mixed with $5 \%$ of copolymer. The three measurement methods are compared: Shear Wave Elastography (SWE), high-frequency rheometer, and hyperfrequency viscoelastic spectroscopy. restricted to a $2 \mathrm{~cm} \times 2 \mathrm{~cm}$ area of white matter. The area of interest is shown in figure 10. The results obtained are shown in figure 12. The data shown are the interquartile range of 40 computations across the $2 \mathrm{~cm}$. From these results viscoelastic parameters were approximated for the power law model. We obtained $(95 \%$ confidence bounds):

$$
\begin{aligned}
G^{\prime}(\omega) & =(1575 \pm 348) \cos \left(\frac{\pi}{2}(0.12 \pm 0.04)\right) \omega^{(0.12 \pm 0.04)} \\
G^{\prime \prime}(\omega) & =(1575 \pm 348) \sin \left(\frac{\pi}{2}(0.12 \pm 0.04)\right) \omega^{(0.12 \pm 0.04)}+(3.79 \pm 0.53)
\end{aligned}
$$
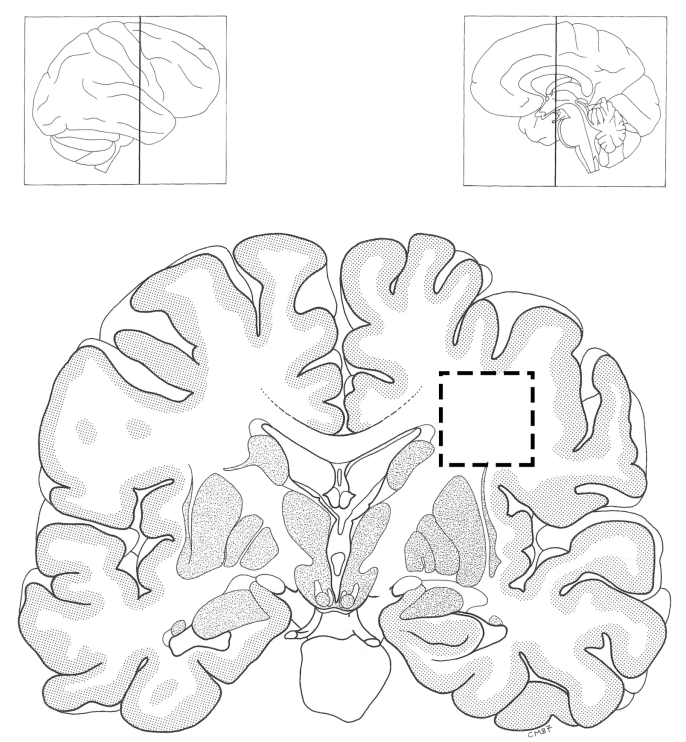

Figure 10: Coronal brain section showing the area measured (dotted square).

The results were compared with those obtained by Nicolle et al. (2005); in their study on porcine brain white matter, they obtained values of $G^{\prime}$ ranging from 
$(2.1 \pm 0.9) \mathrm{kPa}$ to $(16.8 \pm 2.0) \mathrm{kPa}$ when going from $0.1 \mathrm{~Hz}$ to $6310 \mathrm{~Hz}$.

The human brain measurements were correlated to the porcine brain data. Differences can be inputed to the delay between death, extraction and measurement. The $G^{\prime}$ values were globally higher for the the porcine brain, while $G^{\prime \prime}$ were in close proximity, but with a different dispersion behavior.

Using SWE, Chan et al. (2014) revealed a focal area of increased stiffness approximately $1.5 \mathrm{~cm}$ from the brain surface (Fig. 1E) on a 7 year old boy presented with a 5 year history of medication-resistant frontal lobe epilepsy. This area had a mean Youngs modulus (YM) of $74.7 \mathrm{kPa}$, compared to the surrounding gray and white matter, which had a mean YM of $36.2 \mathrm{kPa}$ and $20.8 \mathrm{kPa}$, respectively. If we assume that $E=3 G^{\prime}$, we measure a YM which varies from $6 \mathrm{kPa}$ at $25 \mathrm{~Hz}$ to $12 \mathrm{kPa}$ at $100 \mathrm{~Hz}$. In Chan et al. (2014), the elasticity is measured from the group velocity which corresponds approximately to the shear velocity at the frequency corresponding to the maximum amplitude of the shear wave. Even if we dont have that information our measurements are lower compared to their work. In her thesis Imbault (2017) measured gray matter brain elasticity with intraoperative SWE for tumor classification (meningiomas, low-grade gliomas, high-grade gliomas and metastasis). Chauvet et al. (2015) reported these results. A global mean elasticity of $(7.3 \pm 2.1) \mathrm{kPa}$ was measured on normal brain tissue. These measurements were done in the gray matter with SWE and are in accordance with Kruse et al. (2008) but are also lower than the Chan et al. (2014) results.

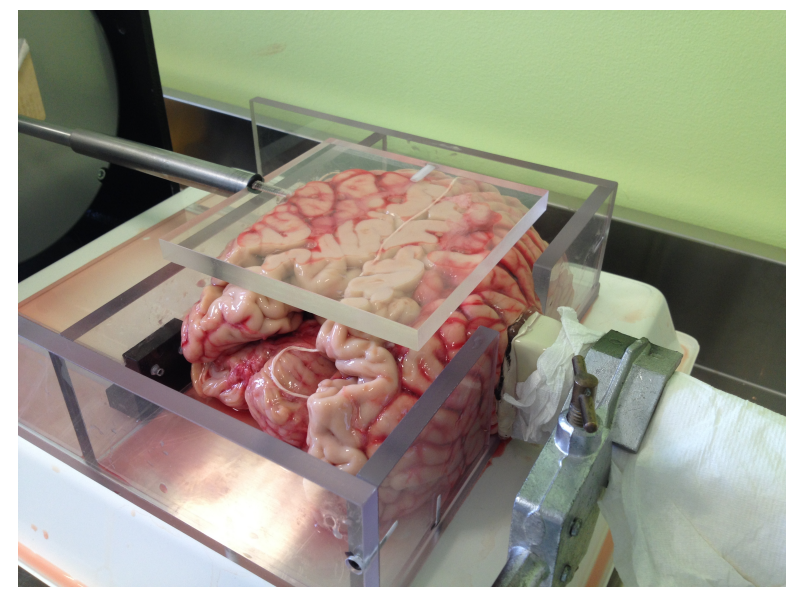

Figure 11: Ex vivo human brain with probe and vibrating plate

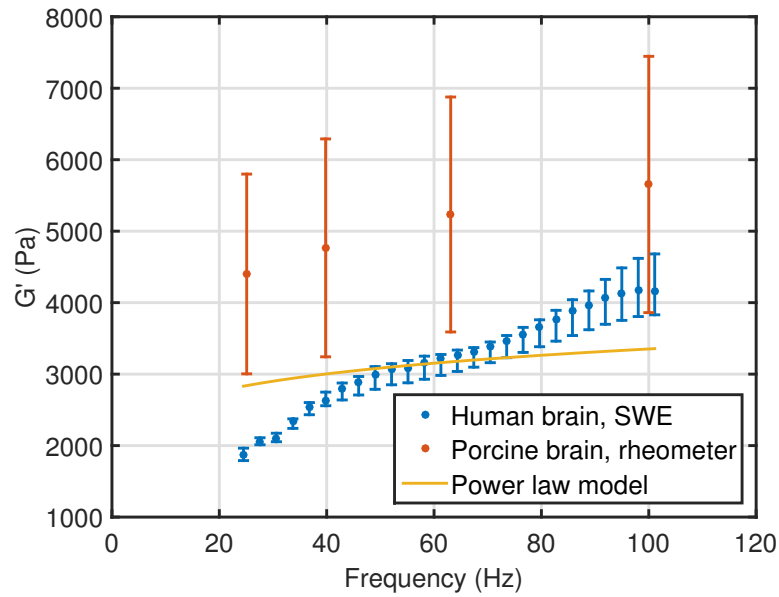

(a) Storage modulus $G^{\prime}$

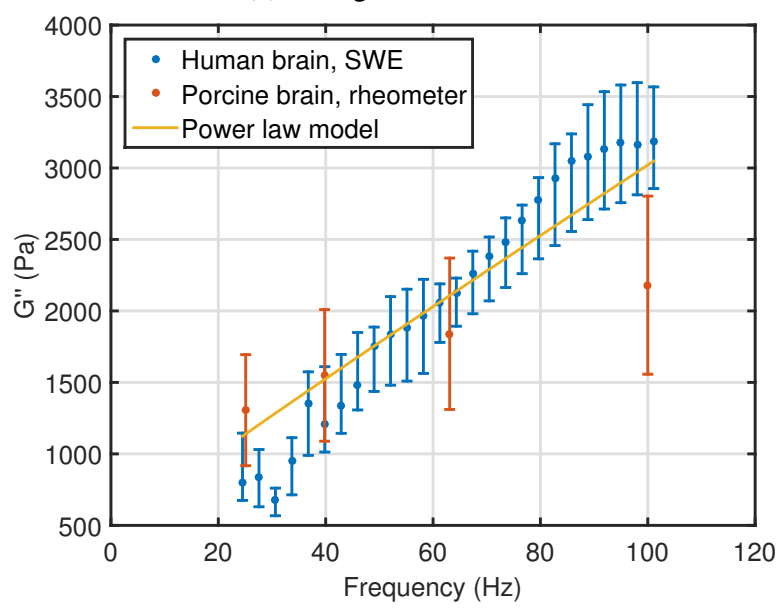

(b) Loss modulus $G^{\prime \prime}$

Figure 12: $G^{\prime}$ and $G^{\prime \prime}$ (storage and loss modulus) for an ex vivo human brain. The data represented is the interquartile range for 40 computations at different depths across the area of interest. The porcine brain data was obtained with a rheometer by Nicolle et al. (2005). A power law fit is represented. 


\section{Conclusion}

The SWE method allowed us to measure the storage modulus dispersion (and thus the shear wave speed dispersion) in two mimicking gels, as well as in ex vivo brain tissue.

The method was successfully tested against two other proven rheology measurement techniques, i.e. hyperfrequency viscoelastic spectroscopy and high-frequency rheometer, in the $20 \mathrm{~Hz}$ to $120 \mathrm{~Hz}$ range. The SWE method was found to provide similar results when measuring $G^{\prime}$, the storage modulus.

Measurement of $G^{\prime \prime}$, the loss modulus, proved to be more challenging. However, when enough care was taken to have at least one wavelength in the observation window the results were then comparable to those obtained with other methods.

These ex vivo measurements show that it is possible to follow the propagation of a shear wave in the brain at a $4 \mathrm{~cm}$ depth, in the the $20 \mathrm{~Hz}$ to $120 \mathrm{~Hz}$ bandwidth. We have enough sensitivity with our method (diffusion of ultrasound from the brain tissue, acquisition SNR and frame rate, Doppler tissue velocity estimation algorithm) to measure the dispersion of $G^{\prime}$ and $G^{\prime \prime}$ with comparable precision to the reference method (rheometer). The main difficulties are to induce plane wave US in the brain through the phase and amplitude aberrating skull with sufficient SNR, to induce measurable shear wave in the brain in vivo, and to estimate the elasticity from these low frequency complex field patterns. Results published by Maitre Maitre et al. (2011) on MRE using the anatomical pathways as waveguides to induce shear wave in the brain are promising. The apparatus developed in this work can be adapted for US brain elastography. Imbault (2017) measures a Young modulus of $(33.1 \pm 5.9) \mathrm{kPa},(23.7 \pm 4.9) \mathrm{kPa},(11.4 \pm 3.6) \mathrm{kPa}$ and $(16.7 \pm 2.5) \mathrm{kPa}$ for meningiomas, low and high grade gliomas respectively, and metastasis with normal brain tissue (gray matter) of $(7.3 \pm 2.1) \mathrm{kPa} 7$, using intraoperative SWE. This biomarker differentiates benign from malignant lesions. Using a modified version of the transient elastography method presented in this study with the guided pressure waves used in brain MRE, we can envision a method to measure brain tissue stiffness in vivo without opening the skull.

\section{Appendix A. Acknowledgments}

We thank Paul Villard-Palluy of the IFSTTAR (Lyon, France) for the rheometer measurements.
We also thank the Anatomy Laboratory of the University of Tours for their help in providing us with $e x$ vivo human brains through the body donation program.

\section{Appendix B. Bibliography}

Bagley, R. L., jun 1983. A Theoretical Basis for the Application of Fractional Calculus to Viscoelasticity. Journal of Rheology 27 (3), 201.

URL https: //dx.doi.org/10.1122/1.549724

Bradley Jr., W. G., Whittemore, A. R., Watanabe, A. S., Davis, S. J., Teresi, L. M., Homyak, M., Bradley Jr., W. G., jan 1991. Association of deep white matter infarction with chronic communicating hydrocephalus: implications regarding the possible origin of normal-pressure hydrocephalus. AJNR Am J Neuroradiol 12 (1), 31-39.

URL http: //www.ncbi.nlm.nih.gov/pubmed/1899515

Catheline, S., Gennisson, J.-L., Delon, G., Fink, M., Sinkus, R., Abouelkaram, S., Culioli, J., 2004. Measurement of viscoelastic properties of homogeneous soft solid using transient elastography: An inverse problem approach. The Journal of the Acoustical Society of America $116(6), 3734$

URL https : //dx.doi.org/10.1121/1.1815075

Chan, H. W., Pressler, R., Uff, C., Gunny, R., St Piers, K., Cross, H., Bamber, J., Dorward, N., Harkness, W., Chakraborty, A., apr 2014. A novel technique of detecting MRI-negative lesion in focal symptomatic epilepsy: Intraoperative ShearWave Elastography. Epilepsia 55 (4), e30-e33.

URL https : //dx.doi.org/10.1111/epi.12562

Chauvet, D., Imbault, M., Capelle, L., Demene, C., Mossad, M., Karachi, C., Boch, A.-L., Gennisson, J.-L., Tanter, M., apr 2015. In Vivo Measurement of Brain Tumor Elasticity Using Intraoperative Shear Wave Elastography. Ultraschall in der Medizin - European Journal of Ultrasound 37 (06), 584-590.

URL https : //dx.doi.org/10.1055/s-0034-1399152

Hadj Henni, A., Schmitt, C., Tremblay, M.-É., Hamdine, M., Heuzey, M.-C., Carreau, P., Cloutier, G., oct 2011. Hyper-frequency viscoelastic spectroscopy of biomaterials. Journal of the mechanical behavior of biomedical materials 4 (7), 1115-22.

URL https://dx.doi.org/10.1016/j.jmbbm.2011.03. 020

Hoeks, A. P., Brands, P. J., Arts, T. G., Reneman, R. S., jan 1994. Subsample volume processing of Doppler ultrasound signals. Ultrasound in medicine \& biology 20 (9), 953-65.

URL https://dx.doi.org/10.1016/0301-5629(94) 90054-X

Huston, J., Murphy, M., Jack, C., Ehman, R., Manduca, A., Felmlee, J., Glaser, K., jul 2011. MR elastography of Alzheimer's disease. Alzheimer's \& Dementia 7 (4), S58-S59.

URL https : //dx.doi .org/10.1016/j.jalz.2011.05.088

Imbault, M., 2017. Imagerie quantitative et fonctionnelle du cerveau humain par ultrasons ultrarapides. Ph.D. thesis, Paris Diderot.

Kruse, S. a., Rose, G. H., Glaser, K. J., Manduca, A., Felmlee, J. P., Jack, C. R., Ehman, R. L., jan 2008. Magnetic resonance elastography of the brain. NeuroImage 39 (1), 231-7.

URL https://dx.doi.org/10.1016/j.neuroimage. 2007. 08.030

Maitre, X., Lemain, E., Sinkus, R., Bruno, L., Darasse, L., 2011. Whole brain MRE with guided pressure waves. In: 19th Meeting of the International Society of Magnetic Resonance in Medicine. Montreal.

Nicolle, S., Lounis, M., Willinger, R., Palierne, J.-F., jan 2005. Shear linear behavior of brain tissue over a large frequency range. 
Biorheology 42 (3), 209-23.

URL http://www.ncbi.nlm.nih.gov/pubmed/15894820

Nicolle, S., Noguer, L., Palierne, J.-F., oct 2013. Shear mechanical properties of the porcine pancreas: experiment and analytical modelling. Journal of the mechanical behavior of biomedical materials $26,90-7$.

URL https://dx.doi.org/10.1016/j.jmbbm.2013.05. 029

Nicolle, S., Vezin, P., Palierne, J., 2010. A strain-hardening bi-power law for the nonlinear behaviour of biological soft tissues. Journal of Biomechanics 43 (5), 927-932.

Oudry, J., Bastard, C., Miette, V., Willinger, R., Sandrin, L., jul 2009. Copolymer-in-oil phantom materials for elastography. Ultrasound in medicine \& biology 35 (7), 1185-97.

URL https://dx.doi.org/10.1016/j.ultrasmedbio. 2009.01.012

Sack, I., Beierbach, B., Hamhaber, U., Klatt, D., Braun, J., mar 2008. Non-invasive measurement of brain viscoelasticity using magnetic resonance elastography. NMR in biomedicine 21 (3), 265-71.

URL https://dx.doi .org/10.1002/nbm.1189

Sandrin, L., Cassereau, D., Fink, M., jan 2004. The role of the coupling term in transient elastography. The Journal of the Acoustical Society of America 115 (1), 73-83.

URL https://dx.doi.org/10.1121/1.1635412

Schiessel, H., Metzler, R., Blumen, A., Nonnenmacher, T. F., dec 1995. Generalized viscoelastic models: their fractional equations with solutions. Journal of Physics A: Mathematical and General 28 (23), 6567-6584

URL https://dx.doi.org/10.1088/0305-4470/28/23/ 012

Szabo, T. L., Wu, J., may 2000. A model for longitudinal and shear wave propagation in viscoelastic media. The Journal of the Acoustical Society of America 107 (5), 2437

URL https://dx.doi.org/10.1121/1.428630

Tanter, M., Fink, M., Robert, B., Sinkus, R., Larrat, B., 2006. A New Rheological Model Based on Fractional Derivatives for Biological Tissues. In: 2006 IEEE Ultrasonics Symposium. IEEE, pp. 10331036

URL https://dx.doi.org/10.1109/ULTSYM.2006.268

Vappou, J., Breton, E., Choquet, P., Willinger, R., Constantinesco, A., oct 2008. Assessment of in vivo and post-mortem mechanical behavior of brain tissue using magnetic resonance elastography. Journal of biomechanics 41 (14), 2954-9.

URL https://dx.doi.org/10.1016/j.jbiomech. 2008 . 07.034

Weaver, J. B., Pattison, A. J., McGarry, M. D., Perreard, I. M., Swienckowski, J. G., Eskey, C. J., Lollis, S. S., Paulsen, K. D., nov 2012. Brain mechanical property measurement using MRE with intrinsic activation. Physics in medicine and biology 57 (22), 7275-87.

URL https://dx.doi.org/10.1088/0031-9155/57/22/ 